\title{
Symptomatic contralateral osteophyte fracture with migration causing lumbar plexopathy during oblique lumbar interbody fusion: illustrative case
}

\author{
Brenton Pennicooke, MD, MS, Jeremy Guinn, BS, BA, and Dean Chou, MD \\ Department of Neurological Surgery, University of California, San Francisco, San Francisco, California
}

BACKGROUND While performing lateral lumbar interbody fusion surgery, one of the surgical goals is to release the contralateral side with a Cobb elevator, allowing distraction of the interbody space. Many times, there are large osteophytes on the contralateral side, and the osteophytes can be split open with the Cobb or blunt instrument. It is extremely rare for the actual osteophyte to break off from the vertebral body into the contralateral psoas muscle and lumbar plexus.

OBSERVATIONS The authors report a case of symptomatic lumbar plexopathy caused by an osteophyte fracture after an oblique lumbar interbody fusion requiring a right-sided anterior approach to excise the bony fragment. They illustrate the case with imaging that the radiologist did not comment on, and they also show a video of the surgical excision of the osteophyte through a right-sided anterior lumbar retroperitoneal approach. The authors also show how the patient had spontaneous right-sided electromyography (EMG) firing before excision of the osteophyte and how the EMG firing resolved after excision.

LESSONS Although the literature is plentiful with regard to ipsilateral approach-related complications, the authors discuss the literature with regard to contralateral complications after minimally invasive lateral lumbar interbody fusion.

https://thejns.org/doi/abs/10.3171/CASE21210

KEYWORDS osteophyte; fracture; plexopathy; lateral lumbar interbody fusion; oblique lumbar interbody fusion

The prepsoas retroperitoneal approach is a minimally invasive approach for lumbar interbody fusion initially described by Mayer in $1997,{ }^{1}$ and it uses the corridor between the psoas and the aorta. It avoids dissection through the psoas and avoids the lumbar plexus compared with the transpsoas approach. A common technique is to pierce the contralateral osteophyte with a Cobb elevator to release the spine and allow disc height restoration. During this maneuver, the osteophyte may fracture; however, these fractured osteophytes are typically nondisplaced and do not cause symptoms. We illustrate a case of a displaced fractured osteophyte causing disabling plexopathy requiring an anterior abdominal approach for surgical excision.

\section{Illustrative Case}

\section{History and Examination}

A 74-year-old woman presented with a 1-year history of right-sided radiculopathy secondary to scoliosis. The patient had exhausted conservative care and wished to have surgery. Her radiographs show dextroscoliosis (Fig. 1). Magnetic resonance imaging (MRI) showed L2-S1 stenosis. She was placed on teriparatide for 3 months before surgery because of osteoporosis.

The patient underwent an L2-S1 oblique lumbar interbody fusion as a first staged surgery. A Cobb elevator was used to pierce the contralateral osteophytes to release the spine in the usual manner. The surgery was otherwise uneventful, and there was no abnormal electromyography (EMG) firing at any level during the entire procedure. Two days later, she underwent L2-to-pelvis posterior instrumentation. Postoperatively, the patient had improvement in her preoperative radiculopathy, but she experienced a new and different pain in her right anterior thigh. This was initially attributed to postoperative inflammation, and the finding of a postoperative MRI scan was negative. An intraoperative O-arm (Medtronic) image demonstrated good position of the screws. However, the patient's pain worsened at home. She began to experience weakness in the right leg.

ABBREVIATIONS ALIF = anterior lumbar interbody fusion; CT = computed tomography; EMG = electromyography; LLIF = lateral lumbar interbody fusion; MRI = magnetic resonance imaging.

INCLUDE WHEN CITING Published July 5, 2021; DOI: 10.3171/CASE21210.

SUBMITTED April 7, 2021. ACCEPTED April 15, 2021.

(c) 2021 The authors, CC BY-NC-ND 4.0 (http://creativecommons.org/licenses/by-nc-nd/4.0/). 

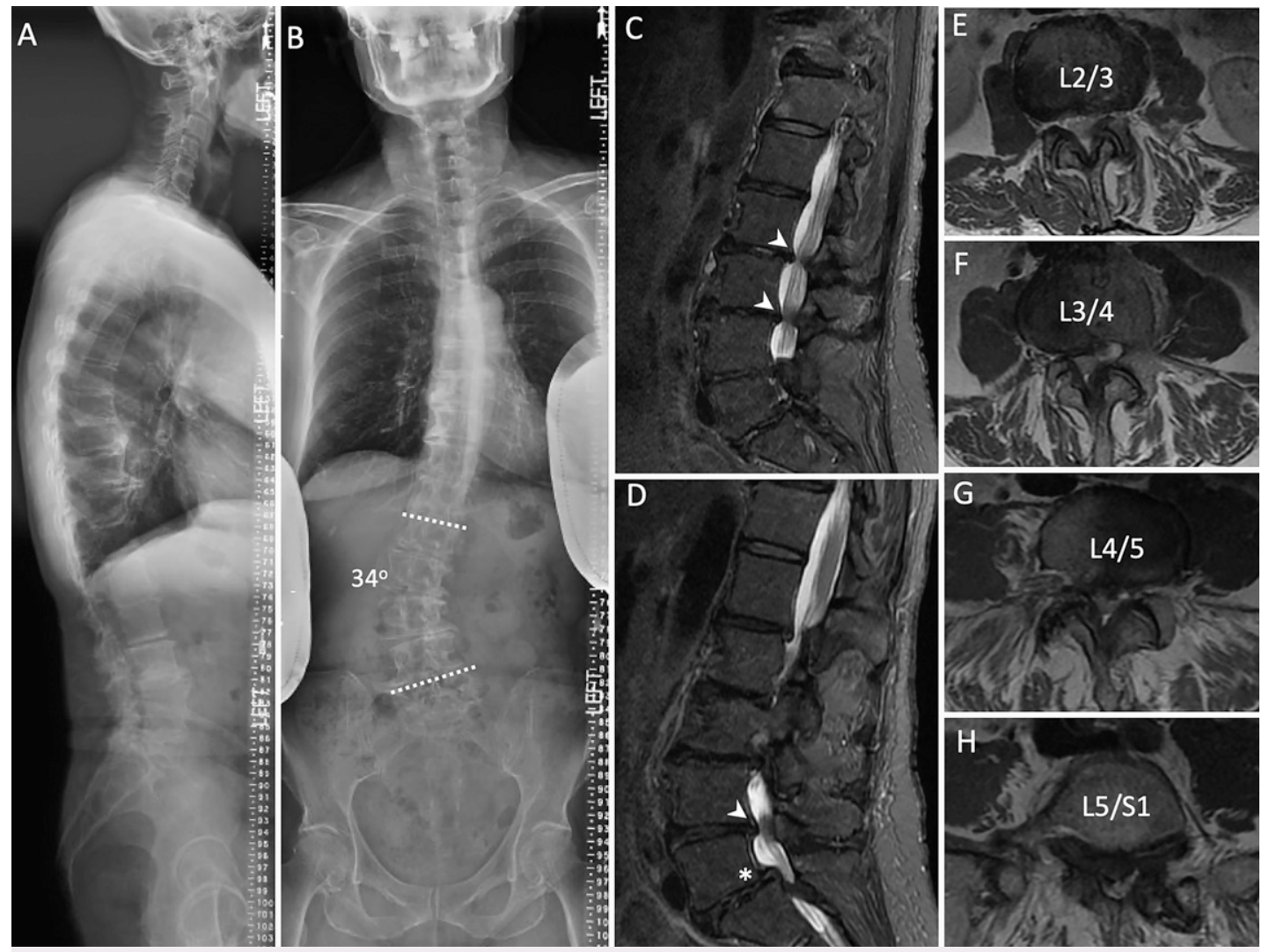

FIG. 1. Preoperative lateral (A) and anteroposterior (B) radiographic images. Preoperative sagittal $T 1$ (C and D) and axial $T 1$ (E-H) MRI scans. Central stenosis can be seen from L2 to L5 (arrowheads) and grade 1 spondylolisthesis (asterisk) in $\mathbf{C}$ and $\mathbf{D}$.

MRI and computed tomography (CT) of the lumbar spine were performed on postoperative day 21. Both studies were read as negative by the radiologist, but detailed review of the CT scan by the surgeons identified that the L4 osteophyte had fractured from the vertebral body into the right psoas muscle, indenting the lumbar plexus (Fig. 2). The CT scan showed a $10.7-\mathrm{mm}$ migration of the fragment into the lumbar plexus. Because of the disabling pain and weakness, the patient was taken to the operating room for retrieval of the migrated $L 4$ osteophyte.

\section{Surgery and EMG Findings}

A standard right-sided anterior lumbar interbody fusion (ALIF) approach to the spine was performed with vascular surgery. The operating microscope was used to identify the osteophyte (Fig. $3 \mathrm{~A}$ ), which was clearly pushing into the right lumbar plexus. The osteophyte was mobile, further accounting for the severe pain with ambulation (Video 1). The fragment was excised, and there was no evidence of compression of the lumbar plexus. The interbody graft was visible after the osteophyte was fully resected (Fig. 3B). Intraoperative EMG showed baseline spontaneous firing and resolution of firing after resection of the osteophyte (Fig. 3C). Postoperatively, the patient's pain had significantly decreased, and at 1 year after surgery, she was hiking 5 miles.

VIDEO 1. Clip showing anterior approach for resection of a symptomatic osteophyte fracture. This video shows the technical steps and considerations for safe removal of an osteophyte protruding into the psoas muscle via an anterior approach. Click here to view.

\section{Discussion \\ Observations}

The lateral lumbar interbody fusion (LLIF) has a risk of nerve injury to the lumbar plexus within the psoas muscles. Generally, this risk is ipsilateral to the approach, with motor and sensory symptoms reported to be between $0 \%$ and $75 \%{ }^{2-10}$ The oblique (or prepsoas) lumbar interbody fusion is reported to have a lower risk of ipsilateral injury to the lumbar plexus than the transpsoas approach because it avoids direct contact with the lumbar plexus. ${ }^{11,12}$ However, in one retrospective multicenter study, 155 patients who underwent the oblique approach had a complication rate of $48.3 \%$. Of these complications, endplate fracture/subsidence $(18.7 \%)$ and transient psoas weakness or thigh numbness $(13.5 \%)$ were the two most common complications. ${ }^{12}$ In contrast, Woods et al. published a study involving 137 oblique lumbar fusion patients and reported no neurological injuries. ${ }^{11}$

Ipsilateral complications after LLIF have been well documented in the literature; however, contralateral complications are less reported. Beckman et al. described 7 cases of symptomatic contralateral psoas hematomas after 3,950 transpsoas LLIFs. ${ }^{13}$ Taher et al. reported contralateral psoas seroma after transpsoas LLIF. ${ }^{14}$ Another study by Taher et al. reported $2.9 \%$ of patients with contralateral motor deficits after LLIF. ${ }^{10}$ Papanastassiou et al. reported $6.3 \%$ of patients who experienced contralateral lower extremity pain after LLIF. ${ }^{15}$ One patient had a displaced endplate fragment, and a laminectomy was performed to remove the fractured bone. Although this complication is similar to the one in our case, their endplate fracture was into the spinal canal, not the contralateral plexus. The endplate fracture 

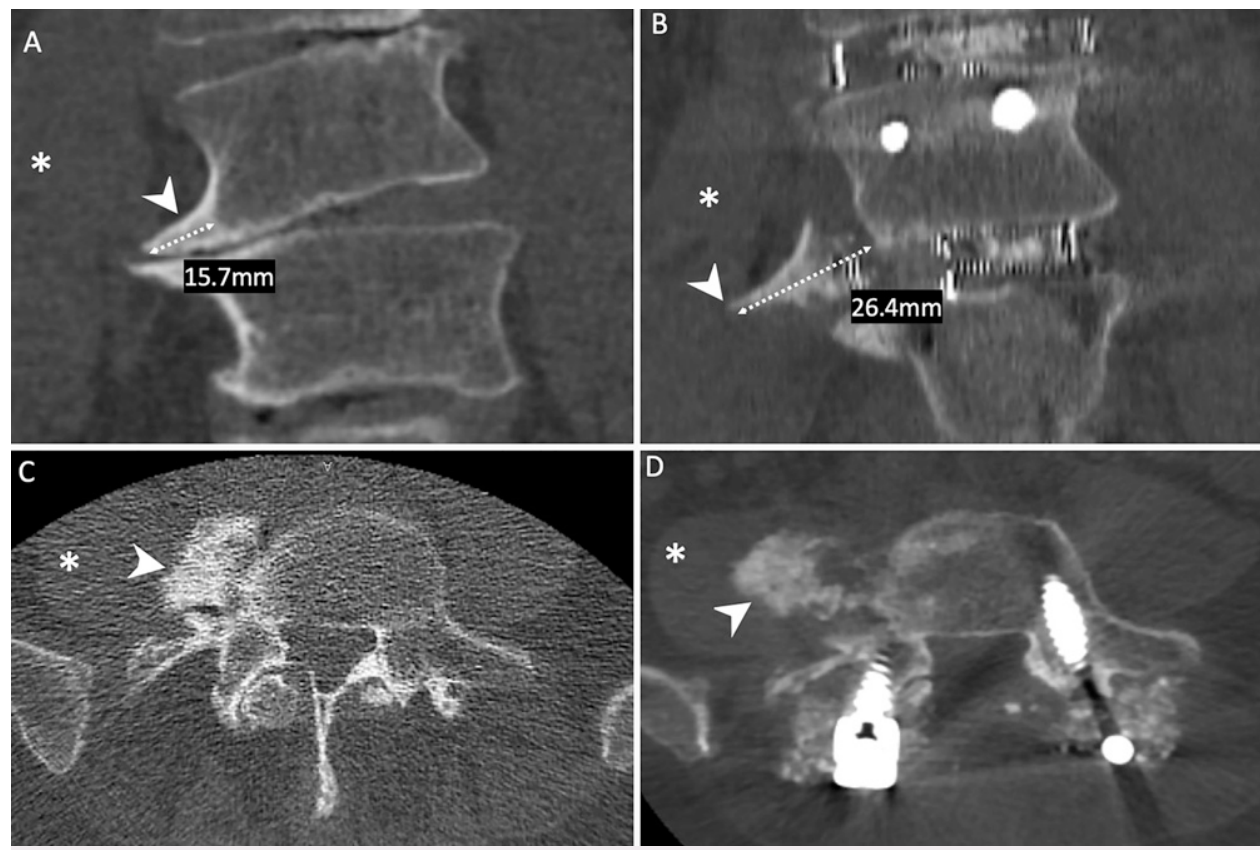

FIG. 2. Initial preoperative CT scan with coronal (A) and axial (C) cuts showing L4 osteophyte (arrowheads) before fracture. Postoperative CT scan with coronal (B) and axial (D) cuts shows the L4 osteophyte fracture protruding into the right psoas muscle (asterisks).

reported in our case was completely located within the psoas muscle, precluding a posterior approach. Papanastassiou et al. concluded that overly breaking the contralateral osteophytes may lead to irritation of the contralateral nerve roots. ${ }^{15}$

Extreme care should be taken when releasing the contralateral osteophyte. Exact parallel trajectory of the instruments from the Cobb elevator, shavers, trials, and implants can help mitigate the risk of fracture. Moreover, complete and thorough release of the contralateral osteophyte, not just the disc space, may help prevent this injury. This is especially important in adult spinal deformity cases in which the osteophytes can be significant and the endplates can be at extreme angles. If a patient has poor bone density, this may also be a factor contributing to possible fracture. ${ }^{15}$ Thus, attention to
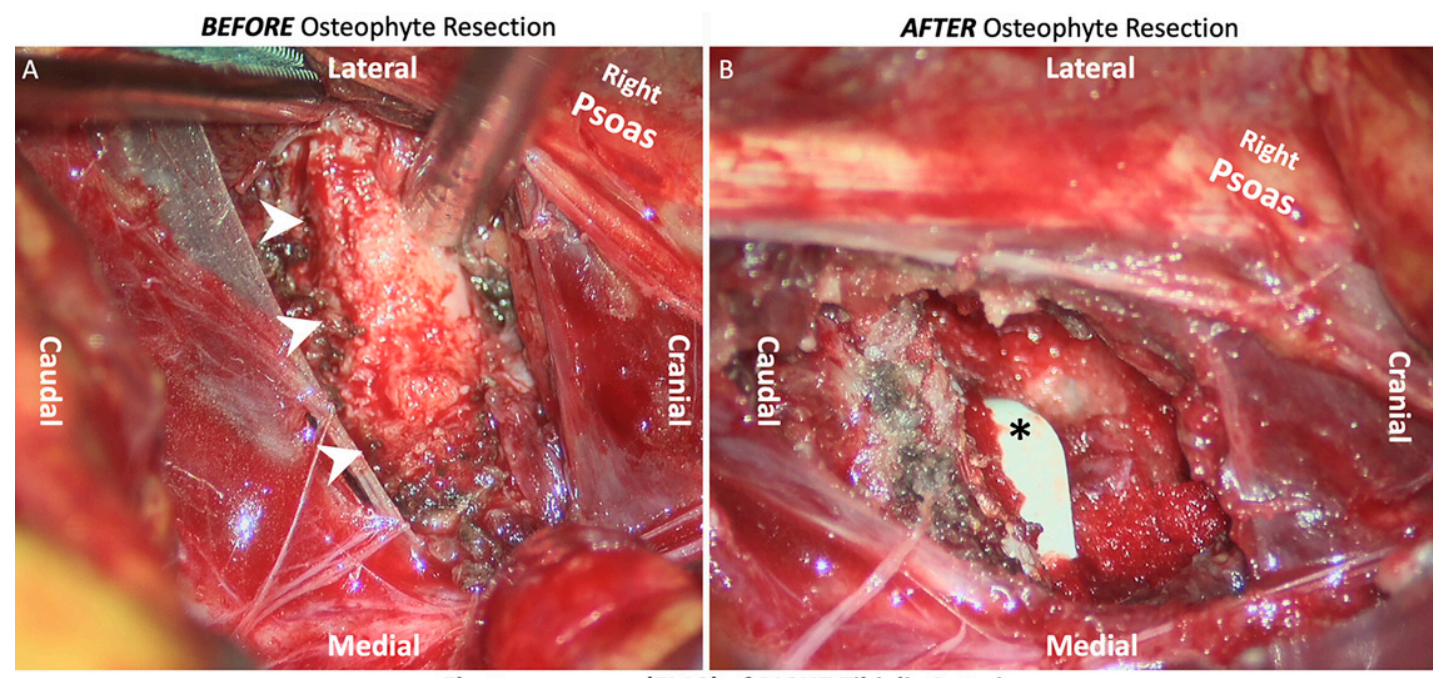

Electromyogram (EMG) of RIGHT Tibialis Anterior

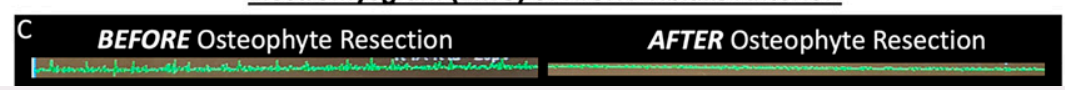

FIG. 3. Intraoperative photographs showing the osteophyte fracture (arrowheads) with the right psoas retracted laterally before resection (A) and the visualized L4-5 cage after resection (asterisk; B). Electromyogram (C) showing irritation of the right $L 4$ nerve root before the osteophyte resection and a reduction of the irritation after the resection. 
technique and execution of the LLIF, especially in cases of deformity and poor bone density, are important to prevent this complication. Because the location of the osteophyte was adjacent to the iliolumbar vein, a standard ALIF approach with vascular surgery was undertaken. To our knowledge, this is the first report of postoperative contralateral plexopathy due to an osteophyte fracture after an oblique lumbar interbody fusion.

\section{Lessons}

When piercing the contralateral osteophyte with a Cobb elevator during lateral interbody fusion, care must be taken that the contralateral osteophyte is not dislodged from the vertebra. If postoperative contralateral symptoms are not readily explained on the basis of routine imaging, consider evaluating the paraspinal region and lumbar plexus as a possible cause.

\section{References}

1. Mayer HM. A new microsurgical technique for minimally invasive anterior lumbar interbody fusion. Spine (Phila $P a$ 1976). 1997;22(6):691-700.

2. Cummock MD, Vanni $S$, Levi $A D$, et al. An analysis of postoperative thigh symptoms after minimally invasive transpsoas lumbar interbody fusion. J Neurosurg Spine. 2011;15(1):11-18.

3. Ahmadian A, Deukmedjian AR, Abel N, et al. Analysis of lumbar plexopathies and nerve injury after lateral retroperitoneal transpsoas approach: diagnostic standardization. J Neurosurg Spine. 2013;18(3):289-297.

4. Lykissas MG, Aichmair A, Hughes AP, et al. Nerve injury after lateral lumbar interbody fusion: a review of 919 treated levels with identification of risk factors. Spine J. 2014;14(5):749-758.

5. Pumberger M, Hughes AP, Huang RR, et al. Neurologic deficit following lateral lumbar interbody fusion. Eur Spine J. 2012;21(6):1192-1199.

6. Aichmair A, Lykissas MG, Girardi FP, et al. An institutional six-year trend analysis of the neurological outcome after lateral lumbar interbody fusion: a 6-year trend analysis of a single institution. Spine (Phila Pa 1976). 2013;38(23):E1483-E1490.

7. Cahill KS, Martinez JL, Wang MY, et al. Motor nerve injuries following the minimally invasive lateral transpsoas approach. J Neurosurg Spine. 2012;17(3):227-231.

8. Uribe JS, Isaacs RE, Youssef JA, et al. Can triggered electromyography monitoring throughout retraction predict postoperative symptomatic neuropraxia after XLIF? Results from a prospective multicenter trial. Eur Spine J. 2015;24(suppl 3):378-385.

9. Le TV, Burkett CJ, Deukmedjian AR, Uribe JS. Postoperative lumbar plexus injury after lumbar retroperitoneal transpsoas minimally invasive lateral interbody fusion. Spine (Phila Pa 1976). 2013; 38(1):E13-E20.
10. Taher F, Hughes AP, Lebl DR, et al. Contralateral motor deficits after lateral lumbar interbody fusion. Spine (Phila Pa 1976). 2013;38(22):1959-1963.

11. Woods KR, Billys JB, Hynes RA. Technical description of oblique lateral interbody fusion at L1-L5 (OLIF25) and at L5-S1 (OLIF51) and evaluation of complication and fusion rates. Spine J. 2017; 17(4):545-553.

12. Abe K, Orita S, Mannoji C, et al. Perioperative complications in 155 patients who underwent oblique lateral interbody fusion surgery: perspectives and indications from a retrospective, multicenter survey. Spine (Phila Pa 1976). 2017;42(1):55-62.

13. Beckman JM, Vincent B, Park MS, et al. Contralateral psoas hematoma after minimally invasive, lateral retroperitoneal transpsoas lumbar interbody fusion: a multicenter review of 3950 lumbar levels. J Neurosurg Spine. 2017;26(1):50-54.

14. Taher F, Lebl DR, Hughes AP, Girardi FP. Contralateral psoas seroma after transpsoas lumbar interbody fusion with bone morphogenetic protein-2 implantation. Spine J. 2013;13(2):e1-e5.

15. Papanastassiou ID, Eleraky M, Vrionis FD. Contralateral femoral nerve compression: an unrecognized complication after extreme lateral interbody fusion (XLIF). J Clin Neurosci. 2011;18(1): 149-151.

\section{Disclosures}

Dr. Chou has served as a consultant for and received royalties from Globus Medical.

\section{Author Contributions}

Conception and design: Pennicooke, Chou. Acquisition of data: Pennicooke, Chou. Analysis and interpretation of data: Pennicooke, Chou. Drafting the article: all authors. Critically revising the article: all authors. Reviewed submitted version of manuscript: all authors. Approved the final version of the manuscript on behalf of all authors: Guinn. Statistical analysis: Pennicooke. Administrative/technical/material support: Pennicooke. Study supervision: Pennicooke.

\section{Supplemental Information \\ Videos}

Video 1. https://vimeo.com/541662736.

\section{Correspondence}

Jeremy Guinn: University of California, San Francisco, CA. jguinn726@ gmail.com. 